\title{
Protée
}

\section{Le rythme et le temps}

\section{Manuel García Martínez}

Volume 27, numéro 1, 1999

La Mort de Molière et des autres

URI : https://id.erudit.org/iderudit/030537ar

DOI : https://doi.org/10.7202/030537ar

Aller au sommaire du numéro

\section{Éditeur(s)}

Département des arts et lettres - Université du Québec à Chicoutimi

\section{ISSN}

0300-3523 (imprimé)

1708-2307 (numérique)

Découvrir la revue

\section{Citer cet article}

Martínez, M. G. (1999). Le rythme et le temps. Protée, 27(1), 33-38.

https://doi.org/10.7202/030537ar

\section{Résumé de l'article}

Le rythme de La Mort de Molière est fondé sur la répétition, la variation et la combinaison d'images et de différents textes. L'absence d'un développement linéaire confère au rythme une place primordiale dans sa perception par le spectateur. Le rythme de cette vidéo présente de surprenantes caractéristiques, comme l'indépendance rythmique des images, des voix, des mouvements des comédiens et de la musique, ou encore une progression construite sur la variation et la combinaison. Cet article analyse ces caractéristiques en relation avec l'expérience du temps.
Ce document est protégé par la loi sur le droit d'auteur. L'utilisation des services d'Érudit (y compris la reproduction) est assujettie à sa politique d'utilisation que vous pouvez consulter en ligne.

https://apropos.erudit.org/fr/usagers/politique-dutilisation/ 


\section{LE RYTHME ET LE TEMPS}

Manuel García Martínez

Le rythme a une grande importance dans La Mort de Molière. Des récurrences dans la succession des plans, dans les voix des comédiens qui disent les divers textes et dans la musique et les bruitages donnent lieu à des structures rythmiques ostensibles. Ces divers rythmes présentent pourtant une indépendance inhabituelle. Par ailleurs, bien que l'enchaînement des séquences n'obéisse à aucune logique fondée sur la consécution-conséquence, les séquences créent l'impression d'un développement. Ce sont ces caractéristiques rythmiques inhabituelles, en relation avec l'impression temporelle qu'elles suscitent, que cet article tente d'analyser concrètement.

Pour cette brève analyse, nous adopterons la définition du rythme proposée par P. Lusson, M. Ronat et J. Lusson: «combinatoire du même et du différent» ${ }^{1}$. L'expérience rythmique est ainsi provoquée avant tout par la répétition et la variation de certains éléments. Nous adopterons, comme constituants élémentaires du rythme, la forme et la durée. Elle dépend aussi, cette expérience rythmique, de la perception des groupements et de l'insertion de ceux-ci dans des structures plus amples, qu'on peut considérer comme les structures supérieures. Cependant, nous n'adopterons pas de structure type, car la variété des structures dans les milieux rythmiques (c'est-à-dire des éléments dans lesquels se produit le rythme: la musique, les voix, les plans) ainsi que leurs différentes natures empêchent leur assimilation ${ }^{2}$.

La caractéristique la plus surprenante dans La Mort de Molière est la présence de rythmes évidents dans la musique, dans les paroles prononcées et dans les images, de rythmes qui ont une indépendance très nette. Cette caractéristique apparaît clairement dès la première image. Un enfant, transportant un tableau, marche avec difficulté: on ne voit que ses pieds. Le mouvement de ces pieds ainsi que le balancement du tableau et son passage par des zones d'ombre et de lumière à quatre reprises constituent les premières structures rythmiques de la vidéo. Le bruit des pas établit un autre rythme, un rythme auditif. Une voix d'enfant récite le premier texte de Heiner Müller, qui proclame l'impossibilité de faire un poème sur Molière tout en présentant l'œuvre comme un poème. Ce rythme de la voix est dissocié des autres rythmes et se poursuit pendant le générique. Cette brève 
image suscite chez le spectateur des attentes du même, peu déterminées, qui demandent à être confirmées. Immédiatement après le générique, l'indépendance des divers rythmes est avérée.

\section{LE RYTHME DES PLANS}

Le rythme des plans est créé par la répétition d'images, souvent de durées très proches, contenant les mêmes éléments, avec des mouvements de caméra et des angles de prise de vue similaires. Ainsi, au début de la vidéo y a-t-il une triple récurrence de travellings latéraux sur des personnages: à deux reprises les visages sont vus de face, puis vus d'en haut. Ces reprises contiennent cependant des variantes: a) dans le nombre de personnages: quatre personnages dans la première image, sept dans la deuxième, trois dans la troisième; b) les deux premières images sont prises sous le même angle, face aux personnages qui se trouvent sur une ligne perpendiculaire au mouvement de la caméra, mais à différentes distances (la seconde est plus éloignée que la première); la troisième image est prise sous un autre angle, surplombant les personnages dont on ne voit plus le visage.

De même, à plusieurs reprises, il y a dans la vidéo des successions rapides de plans, de très courte durée, comme des flashs. Par exemple, les plans 15, 16 et $17^{3}$, montrant successivement une chaise avec des flammes, des pages manuscrites et un fauteuil dans un carré de lumière. Ces plans apparaissent à l'intérieur de la deuxième séquence créée par le récit du Sieur de Grimarest du dernier après-midi de Molière. Ces structures rythmiques sont aisément repérables, car les changements de plans sont inattendus et ces images apparaissent entre des plans beaucoup plus longs où les comédiens effectuent peu de mouvements. L'attention est ramenée au déroulement en soi.

\section{LE RYTHME DES MOUVEMENTS DES PERSONNAGES}

Au sein des images, les mouvements des personnages ne présentent presque aucun rythme, ce qui est exceptionnel. De très rares mouvements, comme les mouvements des mains du père de Molière nettoyant le fauteuil (plan 38), peuvent être considérés comme des rythmes. Jusqu'à l'annonce de la mort de Molière, ces mouvements sont nettement soulignés par l'immobilité qui les précède et qui les suit. Les relations de synchronie entre parole et mouvements, qui constituent l'une des pulsations les plus importantes dans les spectacles de théâtre, disparaissent ici. Cette caractéristique est surtout frappante dans la première partie de la vidéo. La plupart du temps, les personnages demeurent immobiles ou réalisent des gestes ou des mouvements très lents. Se développant sur un fond d'immobilité, les rares mouvements sont séparés par des temps d'arrêt et sont souvent interrompus au milieu de leur développement, dans la phase d'extension: on l'observe par exemple, à plusieurs reprises, dans la scène des acteurs de la commedia dell'arte (plan 24 de la quatrième séquence pendant qu'est lu l'extrait du Dom Juan de Molière). La durée est ainsi représentée par différentes figures qui illustrent sa suspension. Les comédiens ne participent d'un rythme que dans la mesure où, figures immobiles, ils apparaissent d'une façon récurrente dans les plans et peuvent donner lieu à un rythme visuel. Les mouvements de caméra rendent incontournable la perception de ces récurrences. En l'absence de mouvements, les plans deviennent l'unité rythmique du film, et ce en dépit de leurs différentes durées.

\section{LE RYTHME DES VOIX}

Différentes voix récitent des textes de Heiner Müller, Plutarque, Lucrèce, Grimarest, Molière, etc. Chaque texte lu possède un rythme différent. Chaque voix, quant à elle, recourt à un rythme particulier, produisant ainsi une grande variété de débits. Cependant, certains aspects leur sont communs. Toutes les voix utilisent une parole non spontanée proche de la lecture. Dans presque tous les textes, le rythme des syllabes est marqué par une articulation très précise ${ }^{4}$.

Il y a aussi un très grand nombre de pauses, notamment dans les premiers textes: plusieurs groupes de souffle ont un ou deux mots. Le rythme est d'abord fondé sur l'alternance des moments de 
phonation et des pauses, beaucoup plus longues que dans la parole spontanée. Ces nombreuses pauses mettent en relief la parole comme matériau sonore. Alors que la lenteur met en valeur la structure grammaticale, les silences tantôt rassemblent l'expression d'images ou d'idées traditionnellement séparées, tantôt, au contraire, fragmentent l'expression d'une seule image. Cette tendance persiste jusqu'au dernier texte malgré l'accélération très importante du débit phonique.

Dans chaque intervention, il y a des variations dans la prononciation qui reflètent des changements d'intention ou de sentiment de la part du locuteur, sans que le contenu du texte le justifie. Ainsi, dans la vidéo en français, la voix de l'enfant qui prononce le premier texte semble, à un moment donné, contenir un sanglot. Le jeu de l'acteur, toujours présent dans la parole, est alors évident. Ces variations, regroupant des fragments du texte, constituent des séquences rythmiques. De ce fait, dans chaque texte, et par des moyens différents, est créée une parole non spontanée, objet ostensible d'une élaboration artistique. Néanmoins, sauf dans de rares cas ${ }^{5}$, la parole ne possède pas les caractéristiques les plus fréquentes d'une parole théâtrale que sont les allongements de voyelles ou encore les variations mélodiques très marquées. Les courbes mélodiques jouent un rôle rythmique beaucoup moins important qu'au théâtre.

\section{LE RYTHME DE LA MUSIQUE}

La musique composée par Philip Glass accompagne tout le spectacle. Les fragments musicaux coïncident avec certains textes, contribuant à créer diverses séquences; la musique marque également certains moments de transition dans le texte et plus rarement d'un plan à l'autre.

L'analyse détaillée du premier fragment musical de La Mort de Molière permet d'éclairer la logique de constitution des fragments musicaux de la vidéo. À partir d'une structure de base, qui se répète pendant toute la première séquence, l'apparition de nouveaux éléments donne lieu à de nouvelles combinaisons.
Leurs reprises et leurs superpositions forment les fragments musicaux.

Le schéma du premier fragment musical (retranscrit à la fin de cet article) peut être décrit de la façon suivante: le deuxième plan (le travelling latéral sur le haut des visages des trois femmes, après le générique) et le fragment musical commencent simultanément. Le début est constitué par trois sons graves comme des bourdons, semblables à certaines sirènes de bateau. La mesure est de 12/8, soit une mesure de 4 temps avec une division ternaire. Ce bourdon fonctionne comme un appel. Cette série est reprise deux fois. À ce son est ensuite ajouté un autre son simultané, qui se trouve une octave au-dessus, et qui ressemble à celui d'un violoncelle. Après cette exposition, apparaît un arpège qui conclut avec un obstinato. Chaque accord est composé de deux tercets, soit 6 notes, qui sont répétées, pour occuper une mesure. Le thème est composé de quatre mesures. La fin de chaque thème est nettement marquée par l'utilisation de deux accords différents, alors que les mesures précédentes du même thème ne contenaient qu'un seul accord. Appelons "A» cette série de notes. Ces accords constituent la structure qui est reprise dans cette première séquence. La seconde série (B) est une nouvelle disposition de ce même arpège. La ligne mélodique se fonde sur les mêmes accords que la série A, auxquels est superposée une autre série de notes. Cette série est répétée. La série $\mathrm{C}$ est fondée sur une syncope. La dernière mesure de cette série est marquée par quatre accords. La série $\mathrm{D}$, faite d'une musique de flûte, se caractérise par une note longue sur le troisième temps. À partir de ce moment, les séries antérieures sont reprises et combinées entre elles $^{6}$. Cette forme de composition apparaît pendant toute la vidéo. Elle reflète une logique de combinaison semblable à celle des plans.

\section{LA PROGRESSION DE LA MISE EN SCÈNE}

Au cours de cette première séquence s'établissent des attentes concernant l'indépendance du rythme des plans, de la musique et de la parole. En l'absence presque totale de rythme dans les mouvements des 
comédiens, les pulsations sont successivement et parfois simultanément garanties par les plans, la parole et la musique, de façon indépendante. Cette dissociation entraîne, contrairement à la plupart des mises en scène de théâtre, une absence d'ambiguité rythmique; elle a également pour conséquence de retarder l'apparition des attentes à propos des voix et des mouvements, celles-ci étant avant tout fondées sur des relations de simultanéité.

Le déroulement ultérieur confirme, par des caractéristiques similaires, la constitution de séquences. Le plan du fauteuil entouré de vitres réapparaît, marquant nettement le début des séquences. À chaque séquence correspond au moins un texte long et le plus souvent un fragment musical.

À partir des récurrences, une progression s'établit par la transformation graduelle des séquences. Les deux premières contiennent un ou plusieurs plans (ou séries de plans) de même contenu, de durée similaire, ou créés par le même mouvement de caméra: les personnages assis, montrés grâce à trois travellings latéraux, et Molière couché. À partir de la séquence 7, les séquences ont une complexité croissante, avec un plus grand nombre de mouvements de caméra (de plus en plus complexes) et de mouvements chez les comédiens? ${ }^{\text {? }}$.

Les personnages et les objets qui apparaissent dans les plans fixes au début de la vidéo sont repris et associés dans des plans qui révèlent la place qu'ils occupent dans l'espace et que l'on ne pouvait deviner quand ils étaient pris isolément. Les images sont insérées dans des cadres de plus en plus grands jusqu'au plan d'ensemble (plan 61), où apparaît la totalité de l'espace occupé par les éléments montrés avant la séquence 9. À la fin de cette séquence, on verra Molière jetant lui-même au feu les feuilles manuscrites, puis le spectateur entendra l'annonce de la mort de Molière. Ce plan d'ensemble est-il l'image d'un moment de lucidité où, au moment de mourir, apercevant l'ensemble de sa vie - expérience de rappel avant la mort dont parle la psychologie -, Molière décide de brûler son œuvre? En tout cas, la reconstitution de la totalité est niée par la distance et parodiée par la balle qui traverse l'espace. S'agit-il alors d'une affirmation de la validité du fragment et de la séparation? La dernière séquence est faite d'images partielles, qui ne montrent pas l'espace mais semblent plutôt indiquer, par un mouvement rapide de la caméra, l'impossibilité de l'atteindre dans l'échappée des figures hors du cadre, avant l'image unique de la pierre tombale. Tout paraît s'accélérer et se mélanger après l'annonce de la mort: l'impression d'indépendance des rythmes est partiellement amoindrie par la rapidité, les raccords entre les plans deviennent des raccords de jeu, les plans se succèdent sans nouvelle division de séquence, le texte prononcé très rapidement semble un foisonnement du discours avant le silence ultime.

Simultanément et d'une façon paradoxale, alors que la progression est objectivement indiscutable, plusieurs éléments en amoindrissent la perception et le souvenir. Cette impression est provoquée par la suppression des rythmes gestuels et des rythmes vocaux habituels, de même que par la récurrence des mêmes plans sous les mêmes angles de prise de vue et par la musique répétitive. L'attente devient une attente de la récurrence. Par ailleurs, l'absence de liaisons entre les textes, de raccords habituels entre les plans, et l'aspect aléatoire du moment de leur apparition maintiennent l'imprévisibilité du plan suivant. Ainsi, l'insertion des plans dans les séquences (soit l'insertion d'un élément rythmique dans une unité supérieure), qui crée habituellement une attente à la fois plus précise et d'une portée plus importante qu'une image isolée, est considérablement diminuée. Il en est de même de la récurrence de certains plans. Généralement, la reprise d'une même image dans un film sert à montrer la transformation, l'évolution qui a eu lieu, l'écart entre les occurrences. Dans La Mort de Molière cependant, la reprise fréquente et identique d'un plan comme celui du fauteuil vide - pièce de musée, qui anticipe la conséquence de la disparition de Molière - lui fait perdre sa valeur anaphorique. L'image du fauteuil, symbole de la mémoire, coïncide avec la difficulté du spectateur à se rappeler le déroulement antérieur. 


\section{LE RYTHME ET LE TEMPS}

Le rythme suppose une manière d'envisager un déroulement temporel. Il est une position par rapport au temps. Comme dans de nombreux spectacles de Robert Wilson, le rythme et son absence sont construits pour produire chez le spectateur une conscience de la durée à travers une sensation de lenteur. Mais l'expérience du temps est aussi provoquée par d'autres procédés: 1) certaines phrases, certaines expressions des textes, de même que certaines images montrent le passage ou la suspension du temps: la pierre tombale avec sa date, l'enfant et le père, la maladie, le feu consumant les pages écrites, la figure de Molière disparaissant en fumée; 2) par ailleurs, de nombreuses images évoquent potentiellement une temporalité: certains lieux communs, par exemple la figure de Molière-Robert Wilson disparaissant dans le feu, sont des références évidentes; 3) les récurrences confèrent à certaines images une densité temporelle par la valeur anaphorique ou cataphorique qu' elles acquièrent dans le déroulement de la vidéo. Ces procédés sont fréquemment et étroitement imbriqués.

Le rythme de cette vidéo provoque donc une réflexion sur le passage du temps, où un événement la mort - est évoqué à travers une densité temporelle mise en place par la fragmentation de la scène dans les plans et par l'éclatement des textes. Dans un temps chronométriquement court (un peu plus de 47 minutes), est présentée une durée démultipliée, une temporalité cyclique mais ouverte.
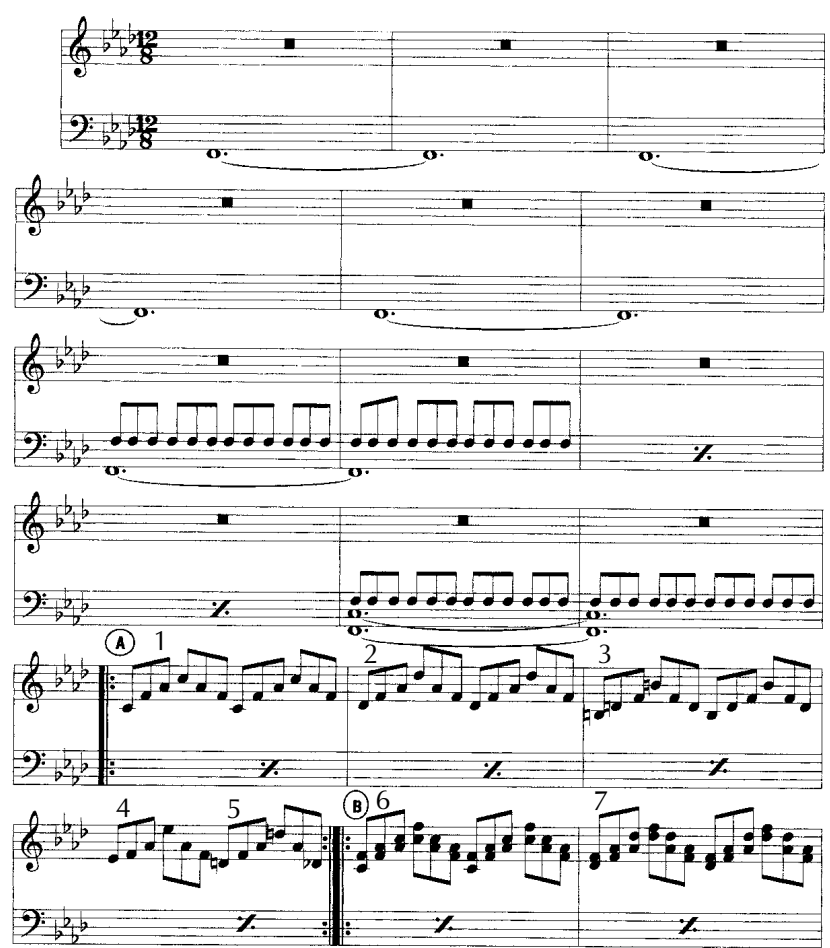
1. fa mineur
3. $\mathrm{sol} 7 \mathrm{e}$
5. si b 7 e
2. ré b Majeur
4. fa 7 e mineur
6. fa mineur
7. ré b Majeur 8. sol 7e
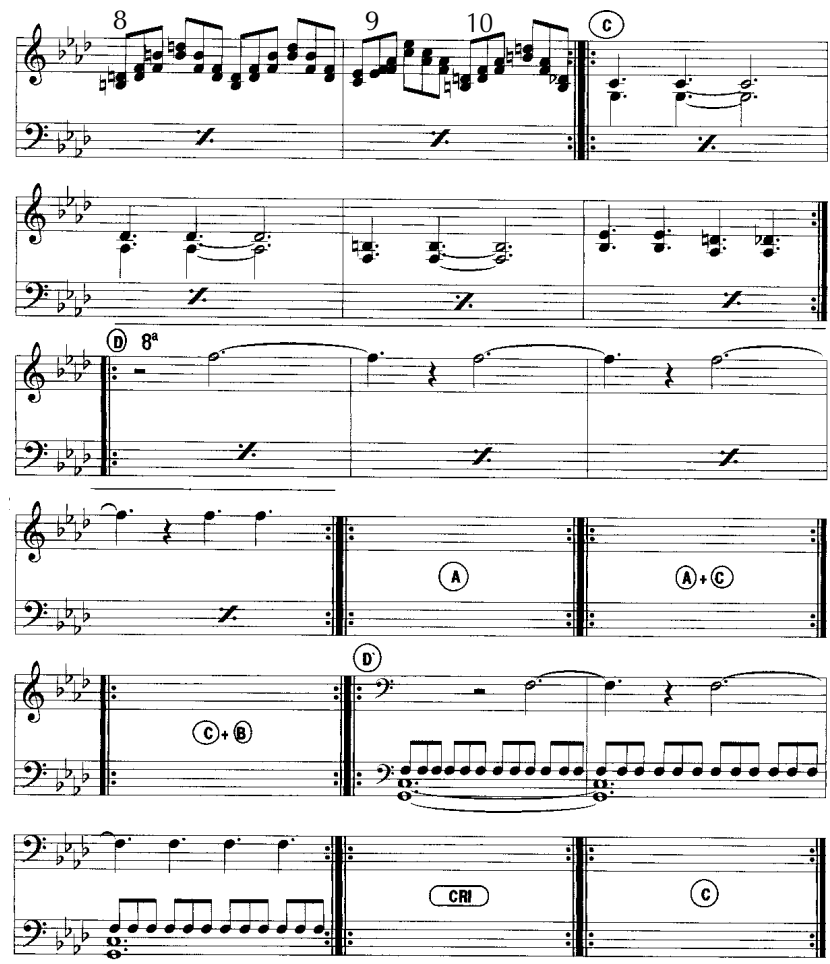

9. do mineur

10. sol $7 \mathrm{e}$ 


\section{NOTES}

1. P. Lusson, M. Ronat et J. Lusson, 1975: 62.

2. M. García Martínez, 1996: c1-c15.

3. Voir le découpage réalisé par $\mathrm{D}$. Bluher.

4. Excepté la fin du texte, que l'on entend pendant que Bulle Ogier, jouant Madeleine, sort un scarabée de sa bouche (plan 60).

5. Comme pendant la lecture du fragment de Dom Juan (plans 23, 24, et 25).

6. La série A est reprise. Puis sont reprises les séries A et C, superposées. Ensuite les séries A, B et $\mathrm{C}$ sont produites simultanément. Puis est reprise la série $D$, mais dans un registre plus grave. Au milieu de cette séquence de plans, Molière pousse un cri, qui correspond à quatre mesures, soit à un thème. Après le cri, la séquence $C$ est reprise puis sont superposées les séquences $\mathrm{C}+\mathrm{A}$, puis $\mathrm{C}+\mathrm{A}+\mathrm{D}$, et $\mathrm{C}+\mathrm{A}+$ $\mathrm{D}+\mathrm{B}$. À ces fragments musicaux s'ajoutent des bruits (des toussotements, des bruits de verre cassé, qui ne correspondent à aucun rythme). Le plan suivant, le plan 12, n'est pas accompagné par de la musique, ce qui contribue à créer une rupture avec les plans antérieurs. 7. Par exemple, dans la séquence 6 , le premier plan rapproché montre le personnage de Molière couché. Suivant une trajectoire latérale complexe, les images montrent successivement les carreaux du sol, une partie du sol éclairée, puis, après un travelling ascendant, la main d'un homme qui nettoie un fauteuil. À ce mouvement succède un mouvement descendant jusqu'au sol, puis à nouveau de bas en haut.

\section{RÉFÉRENCES BIBLIOGRAPHIQUES}

BARBA, E. [1990]: The Secret Art of the Performer, Cardiff, Routledge. Deleuze, G. [1968] : Différence et Répétition, Paris, P.U.F.

FRAISSE, P. [1974]: La Psychologie du rythme, Paris, P.U.F.

GARCÍA MARTÍNEZ, M. [1995]: Réflexions sur la perception du rythme au théâtre, Thèse Nouveau Régime, Université de Paris 8;

[1996]: «Remarques sur le rythme au théâtre", Degrés, n87, automne, c1-c15.

[1998]: "Quelques caractéristiques de la prosodie des comédiens français", Théâtre/Public, no 142-143, 3e trimestre.

Lusson, P., M. RonAt ET J. Lusson [1975]: «Entretien avec M. Ronat,

P. Lusson, J. Roubaud. Le rythme, le formel, le formalisme», Action

Poétique, $\mathrm{n}^{\circ} 62$.

PAVIS, P. [1996]: L'Analyse des spectacles, Paris, Nathan.

Roubaud, J. [1990]: La Vieillesse d'Alexandre, Paris, Ramsay. 